\title{
Transient Response \& Electromagnetic Behaviour of Flexible Bow-Tie Shaped Chip-less RFID Tag for General IoT Applications
}

Muhammad Usman Ali Khan*, Raad Raad, Javad Foroughi

School of Electrical Computer and Telecommunication Engineering, University of Wollongong, Wollongong NSW, 2522, Australia

\begin{tabular}{l} 
A R T I C L E I N F O \\
\hline Article history: \\
Received: 11 June, 2020 \\
Accepted: 23 July, 2020 \\
Online: 12 October, 2020 \\
\hline Keywords: \\
Chipless RFID \\
Bow-tie \\
Polymer Substrate \\
FSS \\
RCS \\
PET \\
SEM \\
Coupling coefficients \\
IoT Applications
\end{tabular}

\begin{abstract}
A B S T R A C T
This paper is an extension of Novel Flexible Chip-less Bow-Tie RFID tag in which we presented the design, testing and fabrication of the tag and compared the results with Octagonal Chipless RFID tag. The chipless RFID tag was designed by using simulation software CST microwave studio and fabricated by using laser etching technique on a flexible polymer substrate Polyethylene Terephthalate (PET). The tag operates at frequency ranging from 8 to $18 \mathrm{GHz}$ uses the Frequency Selective Surface (FSS) approach. A series of experiments are performed to measure the Radar Cross Section (RCS) in an anechoic chamber. The tag design is composed of six concentric Bow-Tie shaped loop resonators with one unitary element. In this paper, we demonstrated the Singularity Expansion Method (SEM) based circuit modelling and the transient behaviour of the RFID tag is performed. The coupling coefficients and the induced currents over the surface of Bow tie shaped rings are evaluated. The maximum read range is evaluated and the BowTie RFID tag is proved to be more accurate and efficient with the variation of distance up to $1.8 \mathrm{~m}$ at $0 \mathrm{dBm}$ which is extendable to $2.14 \mathrm{~m}$ for higher input power. This range is maximum to our knowledge for such a high-frequency range of 8-18GHz. The 4-bits BowTie Chipless RFID tag design is compact and can be deployed commercially for general IoT applications.
\end{abstract}

\section{Introduction}

This paper is an extension of work originally presented in the $19^{\text {th }}$ International Symposium on Communications and Information Technologies (ISCIT) 2019 [1].

The Internet of Things (IoT) relies on smart environments comprises of wireless sensor networks [2] and personal area networks [3], [4] in which one interacts with IoT infrastructure must be uniquely identified. However, one big challenge is a need to commence seamless integration of IoT solutions with surroundings and with people [5]. Flexible materials for various IoT applications require a high level of integrity of components and mechanical robustness with repeated rolling and bending capabilities. In particular, flexible smart fitness watches, RFID tags and wearable sensors are good examples of applications that use flexible material. Besides, Elasticity and stretch-ability of materials are the key properties required by electronic devices that require large and reversible deformation. These bendable devices will also need to be versatile and may require the ability to store

* Corresponding Author: Muhammad Usman Ali Khan, Email:

muak803@uowmail.edu.au

www.astesj.com

https://dx.doi.org/10.25046/aj050592 energy, operate with low power, and integrated with other devices and Internet of Things (IoT) applications [6]-[8].

The growth of wearable technology and the success of wearable devices has been hampered by limits such as the lack of materials and manufacturing techniques for seamless integration with electronic parts [9], the robustness in washing, drying and moulding [10], [11]. To address these challenges flexible polymerbased chipless RFID tag can be a good candidate.

In recent years, the research on wearable wireless communication and flexible devices has increased rapidly due to its usability and applications in personal communication systems [12]. For examples; Flexible antennas and Radio Frequency Identification (RFID) tags are used to keep track of several dailyroutine activities, such as heartbeat and blood pressure measurement, the distance walked, the steps are taken and the calories burnt [13].

RFID Technology is growing rapidly and has significant importance in various medical, industrial and commercial applications [13], [14] such as UHF RFID tags, Chipless RFID 
tags and Near Field Magnetic Coupled tags are being in used in various commercial applications and medical applications [15]. In the traditional RFID technology, the backscattered field from the tags are used to transmit information by using time-variant loading or scatterer modulation [16]-[19] however, this technique requires the physical movement of the object which needs to be coded by using inductive coupling or the impedance modulation. Besides, the conventional RFID tags are active circuit, carrying a small near field chip including a power source and a memory at the centre of the body. Whereas the chipless RFID technology doesn't require memory or a chip to save codes, instead, it uses the physical aspects of the body such as geometry and the shape to send the data [13]. The primary principle of the chipless technology is to get the resonance from the body and to encode the backscattered signal at the receiver, without additional circuitry or communication protocols [20]. Various techniques have been proposed but generally, the chipless RFID technology can be categorised into two groups. The first one is the time domain-based also called time-domain reflectometry-based designs, Surface Acoustic Wave (SAW) is an example of this. The second group is frequency domain-based RFID tags sometimes known as spectral-based tags, Frequency Selective Surface (FSS) is an example of this technique. The SAW-based chipless RFID tags are primarily worked by piezoelectricity, in which Interdigital Transducer (IDT) on the surface of piezoelectric materials are used to convert the electromagnetic wave into the acoustic wave. The acoustic waves generate the identification code while propagating through the metallic surfaces of the tag separated by a certain distance and stored on the tag [21], [22]. The SAW RFID tags are commercially available but some important issues such as the reduction in the size, increase in the read range and the data capacity needs to address 15. Besides, the FSS based frequency domain chipless RFID technique is quite promising, in which an incident electromagnetic wave is completely or partially reflected from the surface of the resonators and matches with the resonance frequency, based on the nature and the shape of the elements. The chipless RFID tags are known as passive tags as no additional power is required to operate, which make these tags promising for general IoT applications.

Passive chipless RFID tags have gained much importance as a good candidate for IoT applications because of no additional circuitry, small size, increased lifetime and the cost-effectiveness [23]. As compared to conventional RFID tags, passive tags have many significant advantages such as they are extremely cheap and can be printed over a piece of paper simple as barcodes. Moreover, the wearable applications in which washing and heating will affect the energy source, the absence of the battery and the electrical circuits make these tags promising [24].

The general structure of a chipless RFID system is an adaptive version of the Ultra-Wide Band (UWB) frequency radar [25], in which an incident electromagnetic wave from a transmitter strikes at the surface of the tag and reflect toward the reader and decoder, see Figure 1. When an incident wave $\mathrm{E}^{\mathrm{i}}$ on the surface of the conductive element, a current is induced at the resonant frequency, $9.2 \mathrm{GHz}$ in the case of chipless Bow-Tie RFID tag, see Figure 1.

In this paper, a 4-bit Bow-tie shaped chipless RFID tag is designed and the transient behaviour by the application of ElectroMagnetic (EM) wave is explained. In this design, the polarization feature of non-periodic bow-tie shaped cells is taken into account to get destructive and constructive interferences. The projection of the incidents wave and reflected field and decoded in terms of bits assigned to Radar Cross Sections (RCS) [26]. The RCS is a parameter to describe the level of scattering of a target object, in this case, a tag is the target object.



Figure 1: Structural diagram of Bow-Tie chip-less RFID system

This paper employs a simple equivalent model of the tag by using SEM-based circuit modelling. Furthermore, the coupling coefficients and the induced currents over the rings are evaluated and transient response of the Bow-Tie RFID tag is analysed. Finally, the maximum read range is calculated which is $1.8 \mathrm{~m}$ for $0 \mathrm{dBm}$ and extendable to $2.14 \mathrm{~m}$ for higher incident power, which is maximum to our knowledge for high frequencies of the range 8 to $18 \mathrm{GHz}$.

The rest of the paper is organised as follows: Section II and Section III presents the tag design and its working principle, the material characterisation and optimised designs. Section IV presents the RCS measurements, which further describes the experimental verification of codes, on body RCS measurements, bending analysis and discussion. Finally, the conclusion is presented in Section V.

\section{Design and Operation of Bow-Tie Chipless RFID Tag}

The Bow-Tie shaped chipless RFID tag proposed in [1] is based on conventional Bow-tie shape which is opted as it has the most intense current flow on the edges of the resonating elements [27], hence cause a more intense backscattered signal. Another reason to opt this design is the fact that it provides more flexibility in designing at higher frequencies unlike octagonal [26], square [28], circular [29] and triangular [30] designs. Moreover, in Bowtie chipless RFID tag the asymmetrical shape of the tag in which 
the distance between the resonators is not constant, it diminished the mutual coupling as compared to the symmetrical surfaces, placed very close to each other [31]. In this paper, a 4-bit Bow-tie chipless RFID tag is proposed and the transient behaviour of the tag is presented. The robust tag is comprised of a periodic pattern of six Bow-tie shaped cells applicable for general IoT and wearable applications.

\subsection{Working Principle}

The resonant frequency of the tag depends on the inductance and the capacitance between the concentric metallic bow-tie shaped elements [32]. Therefore, when an EM wave strikes at the surface of the tag it induces currents and the corresponding capacitance and the inductances are changed. The extent of the reflected or transmitted wave after falling on the surface, depends upon the placement and the geometry of the rings. Aa results, surface currents $\left(\mathrm{J}_{\text {in }}\right)$ and $\left(\mathrm{J}_{\text {out }}\right)$ are induced on the inner and outer bow-tie shaped rings, respectively, as shown in Figure 2.

In this case, the whole structure including all the surrounding bow-tie rings, interact with each other and cause the creation of the standing waves. The standing waves produced the same polarization with an odd multiple of the difference of $\pi$ phase by these surface currents which are approximately equal but opposite in phase. Hence, destructive interference results in backscatter wave (Es) and create a specific frequency response. Figure 2. represents the surface current distribution over the elementary cell and all periodic bow-tie shaped rings which tends to be minimum at the inner side of the tag, at the base and ceiling while maximum and almost constant in all vicinities of the BowTie tag. The area of the tag showing minimum concentration correspond to capacitive behaviour and the area where the surface current is intensive corresponds to the inductive behaviour of the tag, see Figure 2.

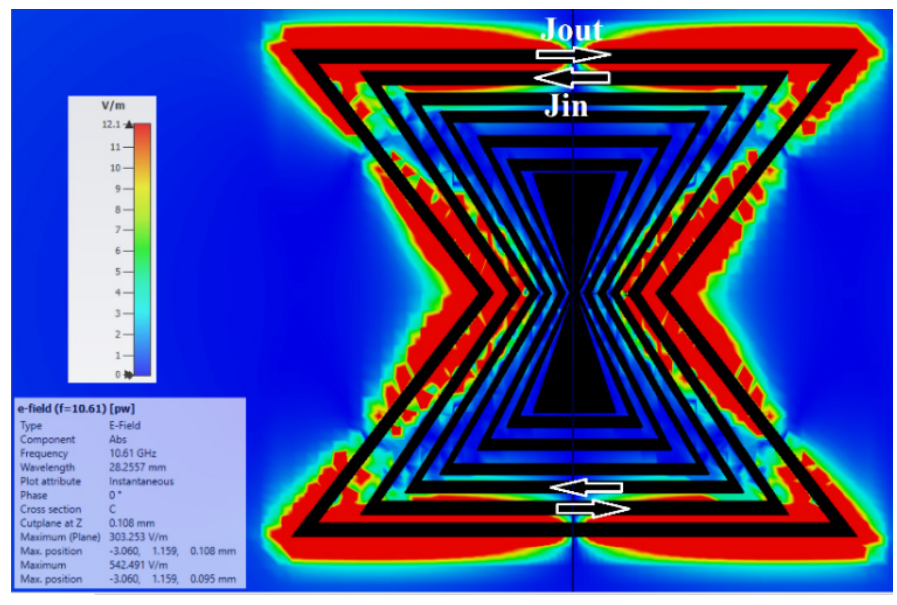

Figure 2: E-Field distribution and the directions of induced surface current (J)

\section{Electromagnetic Behaviour of the Bow-Tie RFID Tag}

The circuit model of the Bow-Tie chipless RFID tag provides insight into the electromagnetic response of the circuit when an incident EM wave strike at the tag and the induced current reradiate the scattered fields. The current induced on the tag can be evaluated in different ways. One method is based on Singularity Expansion Method (SEM) in which the current induced on the tag is expanded in terms of the singularity poles of the tag. The concept of SEM has been widely used in circuit theory for a long time. Later on, this technique was used in evaluating EM response and designing of chipless RFID tags [33],[34],[35].

\subsection{SEM-Based Equivalent Circuit of Chipless RFID Tag}

In this paper, the SEM-based equivalent circuit of the octagonal chipless RFID tag is presented where the solution is evaluated as a collection of poles ( $\mathrm{Sn}$ ), coupling coefficient (Rn) and the entire function $(\mathrm{Fe})$ in the complex frequency domain. The scattering analysis is performed by representing the chipless RFID tag with an equivalent circuit representation, see Fig. For electromagnetic response, the RFID scatterer assumed to be Perfectly Electric Conductor (PEC) in free space. Let's $\mathrm{N}$ be a total number of scatterers and an incident electric field $\mathrm{E}^{\mathrm{i}}$ strike at the surface of the tag and $E^{s}$ are scattered electric field with polarization vectors $\hat{a}_{i}$ and $\hat{a}_{s}$, respectively. The $E^{i}$ causes an induced current $\left(\mathrm{J}_{\mathrm{n}}\right)$ on the surface and the global resonances are demonstrated in the time domain as damped sinusoidal analogous to the Complex Natural Response (CNR) of the scatterer with some weighting residue as coupling coefficients $\left(\mathrm{R}_{N}\right)$. The distance between the two scatterers is optimised in such a way that the coupling between one and the alternative scatterer is very small to be negligible. Hence, the coupling between the two scatterers only at a time and ignored the effects of the others on them.

The SEM-based circuit equivalent model of the octagonal chipless RFID tag is presented in Figure 3. where, $R_{1}, R_{2} \ldots . . R_{N}$, $\mathrm{L}_{1}, \mathrm{~L}_{2}, \ldots . . \mathrm{L}_{\mathrm{N}}$ define the resistances and the inductances and $\mathrm{C}_{1}$, $\mathrm{C}_{2}, \ldots \mathrm{C}_{\mathrm{N}}$ is the inductance between two consecutive scatterers for $\mathrm{N}$ number of resonators.

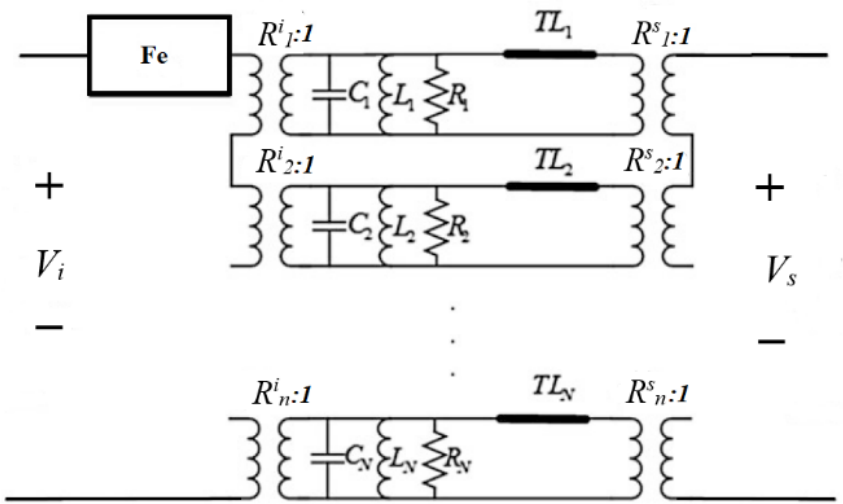

Figure 3: SEM-based equivalent circuit of Bow-Tie RFID Tag with N number of resonators

\subsection{Transient Response of Bow-Tie Chipless RFID Tag}

The $E^{\mathrm{i}}$ coupled to CNR's by coupling coefficients $\mathrm{R}_{1}^{\mathrm{i}}, \mathrm{R}_{2, \ldots}^{\mathrm{i}}$. $\mathrm{R}_{\mathrm{n}}^{\mathrm{i}}$ which depends on the direction and the polarization of incident electric filed, whereas $\mathrm{R}_{1}{ }_{1}, \mathrm{R}_{2, \ldots .}^{\mathrm{i}} \mathrm{R}_{\mathrm{n}}^{\mathrm{i}}$ denotes the coupling coefficients corresponding to $\mathrm{E}^{\mathrm{s}}$. The CNR of the model is presented by a parallel RLC circuit in series with delay line (TL) which designate the turn-on time of CNR, and Fe shows the early time response of the tag. Whereas, the input $\mathrm{Vi}$ and output voltages $\mathrm{Vs}$ are defined at the transmitting and receiving antennas ports, respectively. 
Figure 4. shows the geometry of the Octagonal tag, when $\mathrm{E}^{\mathrm{i}}$ strikes at the surface of the tag and $\mathrm{E}^{\mathrm{s}}$ electric field is scattered with polarization vectors $\hat{a}_{i}$ and $\hat{a}_{\mathrm{s}}$ assuming the incident electric field as

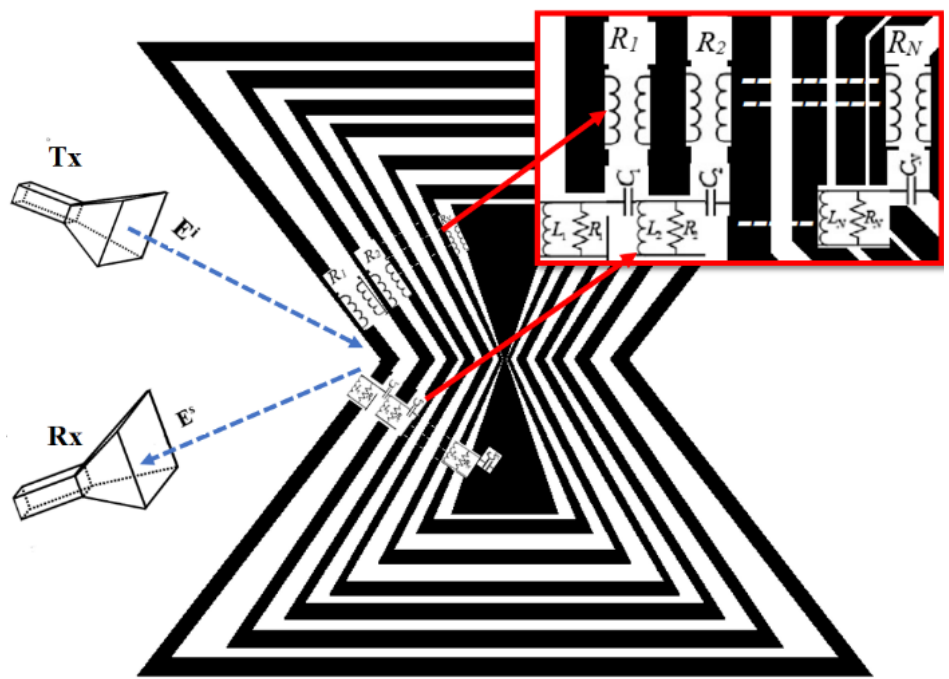

Figure 4: The geometry and the internal circuit modelling of the Bow-Tie RFID Tag Illuminated by an Incident Field Ei

a Dirac-delta function then the transfer function of the tag in the complex s-plane is defined as [15]

$$
H_{t}\left(\hat{a}_{i}, \hat{a}_{s} ; s\right)=\frac{E^{s}(r ; s)}{E^{i}(r ; s)}
$$

After some mathematical manipulation, the transfer function in terms of early-time response $\mathrm{H}_{\mathrm{e}}(\mathrm{s})$ and late time response $\mathrm{H}_{1}(\mathrm{~s})$ with CNR's can be written as [15]

$$
\begin{aligned}
& H(s)=H_{e}(s)+H_{1}(s) \\
& =H_{e}(s)+\sum_{n=1}^{N}\left[\frac{A_{n}}{s-S_{n}}+\frac{A_{n}^{*}}{s-s_{n}{ }^{*}}\right]
\end{aligned}
$$

where

$$
A_{n}=\frac{C}{2}\left(1+j \frac{1+\alpha_{n}}{\omega_{n}}\right)
$$

circuit is described by and CNR's of the

$$
\begin{aligned}
& s_{n}=\alpha_{n}+j \omega_{n} \\
& \alpha_{n}=-\frac{\omega_{0}}{2 Q} \text { and } \omega_{n}=\frac{\omega_{0}}{2} \sqrt{4-\frac{1}{Q^{2}}}
\end{aligned}
$$

where Q represents quality factor which is kept below 0.5 and the RFID first resonator resonates at $f_{1}=\omega / 2 \pi$.

\subsection{Evaluation of Surface Current Distribution (Jn) and the Coupling Coefficients (Rn).}

The current distribution over the first resonator, the outermost octagonal ring is $I_{1}$ and $V_{1}$ is the induced voltage due to incident fields corresponding to the impedance $Z_{1}$ of the resonator then the current distribution over the octagonal scatterers can be evaluated by discretizing the length of the ring into $\mathrm{N}$ number of segments [15] as

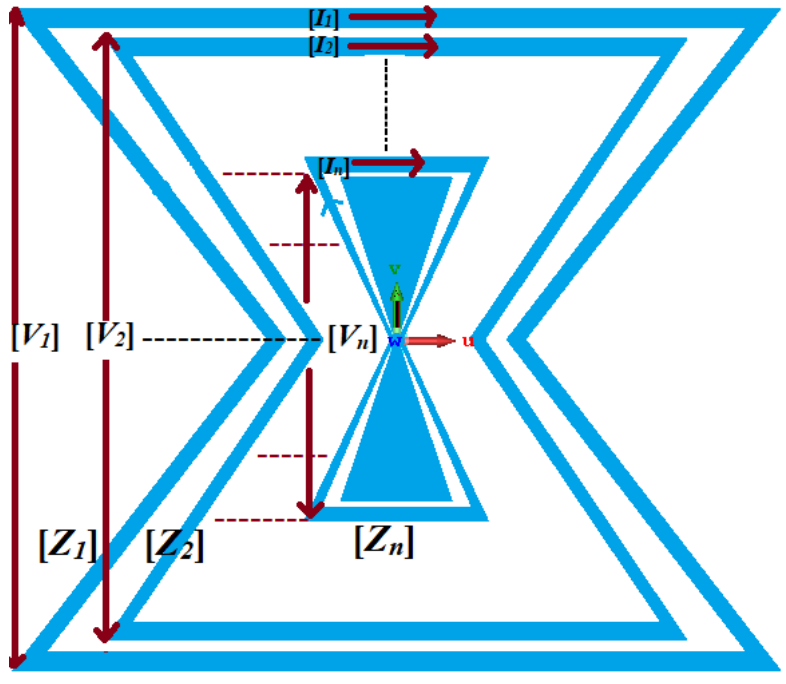

Figure 5: The induced voltages and currents distribution over the surface of the tag

$$
\left[I_{1}\right]=\left[Z_{1}\right]^{-1}\left[V_{1}\right]
$$

where $\left[Z_{1}\right]$ corresponds to $\mathrm{N} \times \mathrm{N}$ matrix referred to as system impedance matrix, $\left[I_{1}\right]$ and $\left[V_{1}\right]$ are the response vector of $\mathrm{N} \times 1$ corressponding to the incident field, see Figure 5 .

The CNR's for the first scatterer is calculated from

$$
\begin{aligned}
& {\left[\mathrm{Z}_{1}\left(\mathrm{~S}_{\mathrm{n}}\right)\right]\left[\mathrm{I}_{1}\left(\mathrm{~S}_{\mathrm{n}}\right)\right]=0} \\
& \Delta_{1}\left(\mathrm{~S}_{\mathrm{n}}\right)=\operatorname{det}\left[\mathrm{Z}_{1}\left(\mathrm{~S}_{\mathrm{n}}\right)\right]=0
\end{aligned}
$$

The CNR's for the first resonator is obtained by expending $\Delta_{1}\left(S_{n}\right)$ in complex Taylor series.

$$
\Delta_{1}\left(\mathrm{~S}_{\mathrm{n}}\right)=\Delta_{1}\left(\mathrm{~S}_{0}\right)+\Delta_{1}{ }^{\prime}\left(\mathrm{s}_{0}\right)\left(\mathrm{s}-\mathrm{S}_{0}\right)+\ldots=0
$$

By keeping the first two terms of the equation and ignoring the rest of values the $\mathrm{CNR}, \mathrm{s}_{\mathrm{n}}$, is obtained from

$$
s_{n}=s_{0}-\frac{\Delta 1(s 0)}{\Delta 1^{\prime}(s 0)}
$$

where $\mathrm{s}_{0}$ is the initial guess of resonant frequency, by repeating this procedure more accurate values can be evaluated for the first scatterer. For Bow-Tie design, the first resonance was calculated at approximately $9.2 \mathrm{GHz}$.

The equation (5) is modified to get current distribution for the first resonator of octagonal chipless RFID tag as

$$
[I]=\sum_{n} \frac{R_{n}}{S-S_{n}}\left[J_{n}\right]
$$

where $R_{n}$ the residue of nth pole and $J_{n}$ is the surface current distribution over the surface of the scatterer, which is evaluated in the next section. Also, The time-domain response can be obtained by applying inverse Laplace transform to (9). 
To study the mechanism of scattering by a 4-bit octagonal chipless RFID tag, the surrounding medium is supposed as a free space with permittivity $\varepsilon_{0}$ and permittivity $\mu_{0}$, the scatterer is PEC and the thickness of the tag is very small hence considered to be zero. Consider an incident field $\mathrm{E}^{\mathrm{i}}$ strikes and the induced current over the surface of the tag is $\left(\mathrm{J}_{\mathrm{n}}\right)$ then the scattered field $\mathrm{E}^{\mathrm{s}}$ from the tag is either obtained from Electric Field Integral Equation (EFIE) or Magnetic Field Integral Equation (MFIE) [36]. The EFIE is used which is represented in Laplace form [37].

$$
E^{s}(r ; s)=\mu s \iint_{A}\left[\left(\vec{I}-\frac{1}{k^{2}} \nabla \nabla\right) G_{0}\left(r, r^{\prime} ; s\right)\right] . J\left(r, r^{\prime} ; s\right) d S^{\prime}
$$

where A represents the surface area of the octagonal chipless RFID tag, $\vec{I}=\hat{x} \hat{x}+\hat{y} \hat{y}+\hat{z} \hat{z}$, $\mathrm{k}$ represents the propagation constant $\mathrm{k}=\mathrm{s} / \mathrm{c}$ in the complex frequency domain and $\mathrm{G}_{0}$ defines the scalar Green's function in the free space.

$$
G_{0}\left(r, r^{\prime} ; s\right)=\frac{e^{-j k\left|r-r^{\prime}\right|}}{4 \pi\left|r-r^{\prime}\right|}
$$

The coupling coefficients are then evaluated by using the equations

$$
R_{n}\left(s_{n}\right)=-\frac{\left\langle J_{n}(r), E_{t}^{i}\left(r ; s_{n}\right)\right\rangle_{r}}{\left\langle\left\langle J_{n}(r),\left\langle\left.\frac{\partial \vec{G}\left(r, r^{\prime} ; s\right)}{\partial s}\right|_{s=s_{n}}, J_{n}\left(r^{\prime}\right)\right\rangle_{r^{\prime}}\right\rangle_{r}\right.}
$$

This equation shows that the coupling coefficient depends upon $\mathrm{E}^{\mathrm{i}}$ and natural modes $J_{n}(r)$ at the resonant frequency.

$$
\left\langle J_{n}(r), E_{t}^{i}\left(r ; s_{n}\right)\right\rangle_{r}=0
$$

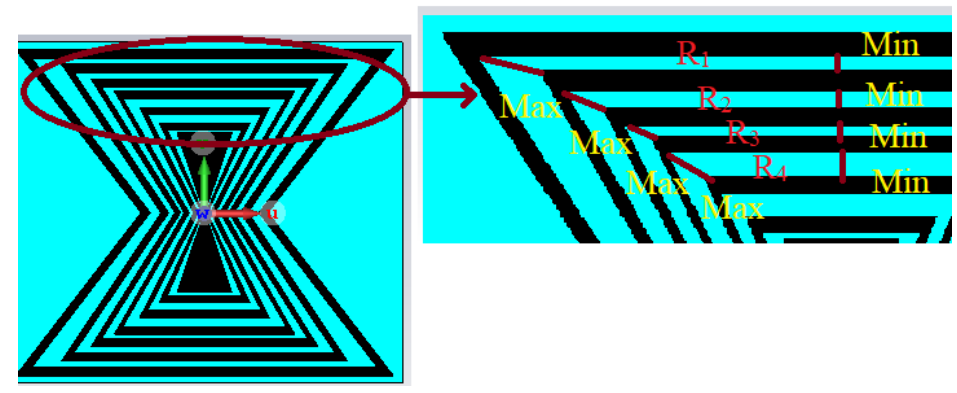

Figure 6: The variation of the distances between consecutive Bow-Tie shaped rings

The evaluation of coupling coefficients $\left(R_{1}\right.$ to $\left.R_{4}\right)$ was not easy in the case of chipless Bow-tie RFID. This is because the distance between the two consecutive bow-tie shaped rings is not constant and hence mutual coupling varies, see Figure 6. Therefore, coupling coefficients are evaluated for the values of the maximum and the minimum distances between two consecutive rings at corresponding resonance, see Table 1 . The values of coupling coefficients between the first two consecutive rings are -0.010 and -0.021 calculated for the maximum and the minimum spacing of
$1.2 \mathrm{~mm}$ and $0.23 \mathrm{~mm}$, respectively for $9.2 \mathrm{GHz}$ frequency, see Table 1. Similarly, all values are calculated for corresponding resonant frequencies of $11.3,14$ and $16.9 \mathrm{GHz}$. The maximum value of $\mathrm{R}_{n}$ from the table is -0.021 which is a sufficiently low value that assures reliable reading of the coded information. To reduce the mutual coupling the average spacing between the resonators can be increased, therefore the size of the tag would be significantly increased.

Table 1: Coupling Coefficients for Corresponding Resonances

\begin{tabular}{|c|c|c|c|c|c|}
\hline $\mathbf{n}$ & $\begin{array}{c}\text { Resonant } \\
\text { Frequency } \\
\mathbf{F}_{\mathbf{0 n}}(\mathbf{G H z})\end{array}$ & \multicolumn{2}{|c|}{$\begin{array}{c}\text { Spacing } \\
\text { Between } \\
\text { Resonators } \\
\text { Min to Max } \\
\mathbf{( m m )}\end{array}$} & $\begin{array}{c}\text { Coupling } \\
\text { Coefficients } \\
\text { Minimum } \\
\mathbf{R n}\end{array}$ & $\begin{array}{c}\text { Coupling } \\
\text { Coefficients } \\
\text { Maximum } \\
\mathbf{R n}\end{array}$ \\
\cline { 3 - 4 } & $\mathbf{D}_{\min }$ & $\mathbf{D}_{\max }$ & & \\
\hline 1 & 9.2 & 0.23 & 1.2 & -0.010 & -0.021 \\
\hline 2 & 11.3 & 0.35 & 0.9 & -0.012 & -0.017 \\
\hline 3 & 14 & 0.17 & 0.6 & -0.009 & -0.014 \\
\hline 4 & 16.9 & 0.52 & 1.1 & -0.014 & -0.020 \\
\hline
\end{tabular}

\section{Flexibility and Bending Analysis}

The Bow-Tie tag is designed on $13.44 \times 11.56 \mathrm{~mm} 2$ PET substrate of $70 \mu \mathrm{m}$ thickness with conductive gold rings varied in thickness [1].



(b)

Figure 7: Chipless Bow-Tie RFID tags a) Experimental set-up in chamber b) Bending aspects of fabricated Chip-less RFID Tags at $27 \mathrm{~mm}$ and $14 \mathrm{~mm}$

\subsection{Experimental Setup and RCS Results}

The chipless Bow-tie RFID tag is printed on a PET substrate by using Universal Laser Systems (ULS). The measurements of 


\section{Radar Cross Section RCS of Bow-Tie Chipless RFID Tag}



the RCS obtained by the reflections from the tag are analysed by Vector Network Analyser (VNA). For this purpose, Keysigth VNA series E5063A is used with two similar horn antennas operating at $4-18 \mathrm{GHz}$ frequencies. To conduct the measurement, the testing is performed in the FRANKONIA anechoic chamber, having a frequency range of $30 \mathrm{MHz}$ to $40 \mathrm{GHz}$. The compact Bow-Tie RFID tag design is highly flexible and the bit codes are successfully obtained up to the bending radius of $14 \mathrm{~mm}$ [38]. The Bow-Tie RFID tag undergoes bending for the following cases of curvatures, flat, $14 \mathrm{~mm}$ and $27 \mathrm{~mm}$, see Figure 7 (b).

The Passive Bow-tie RFID tag is placed in the horizontal $(\mathrm{H})$ position to perform the bending analysis for the following cases flat, $14 \mathrm{~mm}$ and $27 \mathrm{~mm}$. All the testing was performed in the Frankonia anechoic chamber. One of the impacts of bending is the signal strength degradation of the backscattered waves and the significant shift in the resonant frequency. In other words $|\mathrm{RCS}|$ is lowered while tag undergoes bending. In this case, since the distance of the tag from the reader, is kept very small about $12 \mathrm{~cm}$ from the transmitter, so a very small reduction in the signal strength is obtained at $0 \mathrm{dBm}$ power. However, the percentage shift in the resonant frequency is quite prominent especially at a higher level of bending at $14 \mathrm{~mm}$, see Figure 8 .

Figure 8. depicts the impact of bending on the RCS obtained in the chamber. A shift of $4 \%$ from the resonant frequency is obtained, when a PET substrate-based chipless Bow-tie RFID tag undergoes bending curvature $27 \mathrm{~mm}$ for the lower range of the frequencies $8-14 \mathrm{GHz}$, whereas, more than $10 \%$ shift is observed for higher range of frequency up to $18 \mathrm{GHz}$. Hereafter, there is no noticeable reduction of the signal strength is observed because of the small distance of tag from the reader. Hence, the experimental results depict that the RCS obtained and encode in frequency signature, from the bent chip-less RFID tag fabricated on the flexible PET substrate.

\section{Maximum Read Range Estimation}

This section provides the maximum read range of BowTie Chipless RFID tag. The complex RCS feature $\left(\sigma^{\text {Tag }}\right)$ of octagonal chipless RFID designs can be obtained by the following equations which are described in [39].

$$
\sigma^{T a g}=\left[\frac{S_{21}^{T a g}-S_{21}^{N o-T a g}}{S_{21}^{R e f}-S_{21}^{N O-T a g}}\right]^{2} \sigma^{\text {Ref }}
$$

Where $S_{21}^{N o-T a g}$ is associated with the measurement taken in the empty chamber, $S_{21}^{R e f}$ corresponds to reference value taken from define setup which could be any conductive surface, In this case, a copper square of $50 \mathrm{~mm} \times 50 \mathrm{~mm}$ is taken. $\sigma^{R e f}$ is associated with the simulation results for the same reference. The results achieved for all the tags show a good agreement between the measured and simulated designs.

The radar equation is used [40] to evaluate the maximum read range of the RFID tag. For this, numerically calculated RCS are considered by using the equation is follows:

$$
=\sqrt[4]{\frac{G_{T} G_{R} \lambda^{2} \sigma^{\text {Tag }} P_{T}}{(4 \pi)^{3} P_{\text {min }}}}
$$

Where $G_{T}$ and $G_{R}$ are the gains of the transmitting and receiving horn antennas, $P_{T}$ is the power transmitted by VNA to measure RCS, $\lambda$ is the wavelength. $P_{\min }$ is the minimum power receive by receiving antenna and $\sigma^{\text {Tag }}$ is the experimental values of RCS obtained from (14). For maximum read range calculations $\sigma^{T a g}$ is taken for $1111 \mathrm{bit}$ codes, see Figure 8. flat case. The average RCS is obtained from a series of experiments [1] is around $0.02 \mathrm{~m}^{2}$. The maximum read range is evaluated for $-10,-3$ and $0 \mathrm{dBm}$ transmitted power from VNA and the maximum range observed is $1.80 \mathrm{~m}$ and extendable to $2.15 \mathrm{~m}$ for $3 \mathrm{dBm}$ power. Hence, a robust Bow-tie chipless RFID tag with transient analysis 
is successfully designed fabricated and tested, which is applicable for the IoT applications and the read range of the tag and bit capacity can be increased in future by using the asymmetrical property of the tag.

Table 2: Maximum Read Range Estimation

\begin{tabular}{|c|c|c|c|c|c|}
\hline$P_{\min }(d B)$ & $\begin{array}{l}G_{T}(d B i) \\
G_{R}(d B i)\end{array}$ & $\begin{array}{c}\lambda^{2} \\
(\mathrm{~m})\end{array}$ & $\begin{array}{l}\sigma^{T a g} \\
\left(m^{2}\right)\end{array}$ & $\begin{array}{c}\mathbf{P}_{\mathbf{T}} \\
(\mathbf{d B})\end{array}$ & $\begin{array}{c}\mathbf{R} \\
\text { (m) }\end{array}$ \\
\hline \multirow{4}{*}{-70} & \multirow{4}{*}{10} & \multirow{4}{*}{$\begin{array}{c}1.06 \mathrm{x} \\
10^{-3}\end{array}$} & \multirow{4}{*}{0.02} & -10 & 1.01 \\
\hline & & & & -3 & 1.52 \\
\hline & & & & 0 & 1.80 \\
\hline & & & & 3 & 2.15 \\
\hline
\end{tabular}

\section{Conclusion}

This paper is an extension of Novel Bow-Tie Chipless RFID tag which studies SEM-based circuit modelling of the bow-tieshaped tag. The electromagnetic response of the tag is presented and coupling coefficients are obtained by transient analysis. The behaviour of the RFID tag is completely studied and experimentally verified for different patterns of bitstreams measured in terms of RCS. The coupling coefficients measured in this analysis evaluated for the values of the maximum and the minimum distances between two consecutive rings at corresponding resonance of 9.2, 11.3, 14 and $16.9 \mathrm{GHz}$. The maximum value of $R_{n}$ from the table is -0.021 which is a adequately low value that would not impact the reliable coding of the information. Furthermore, the bending on Flexible Chip-less Bow-Tie RFID tag operating within the range of $8-18 \mathrm{GHz}$ are described up to the maximum bend of $14 \mathrm{~mm}$ and the information stored on a bent chip-less RFID tag made of PET is successfully recovered. Finally, the maximum read range for the RFID tag is evaluated which is $1.8 \mathrm{~m}$ for $0 \mathrm{dBm}$ power but extendable to $2.15 \mathrm{~m}$ for higher powers. This range is maximum to our knowledge for such a high-frequency range of $8-18 \mathrm{GHz}$.

\section{Conflict of Interest}

The authors declare no conflict of interest.

\section{Acknowledgement}

I would like to acknowledge the great contribution of my research colleague Panagiotis Ioannis Theoharis, for helping me in the measurements and the fabrications of the tags.

\section{References}

[1] M.U.A. Khan, R. Raad, J. Foroughi, F.E. Tubbal, J. Xi, "Novel Bow-Tie Chip-less RFID Tag for Wearable Applications," in 2019 19th International Symposium on Communications and Information Technologies (ISCIT), IEEE: 10-13, 2019, https://doi.org/10.1109/iscit.2019.8905208.

[2] M. Younan, E.H. Houssein, M. Elhoseny, A.A. Ali, "Challenges and recommended technologies for the industrial internet of things: A comprehensive review," Measurement, 151, 107198, 2020, https://doi.org/10.1016/j.measurement.2019.107198.

[3] A. Čolaković, M. Hadžialić, "Internet of Things (IoT): A review of enabling technologies, challenges, and open research issues," Computer Networks, 144, 17-39, 2018, https://doi.org/10.1016/j.comnet.2018.07.017

[4] E. Jovanov, "Wearables Meet IoT: Synergistic Personal Area Networks (SPANs)," Sensors, 19(19), 4295, 2019, https://doi.org/10.3390/s19194295

[5] T.M. Fernández-Caramés, P. Fraga-Lamas, "Towards the Internet of smart clothing: A review on IoT wearables and garments for creating intelligent connected e-textiles,” Electronics, 7(12), 405, 2018, 2018. https://doi.org/10.3390/electronics7120405

[6] Y. Leterrier, "Mechanics of curvature and strain in flexible organic electronic devices," Handbook of Flexible Organic Electronics: Materials, Manufacturing and Applications, 1, 2014, https://doi.org/10.1016/b978-178242-035-4.00001-4

[7] N.L. Pira, "Smart integrated systems and circuits using flexible organic electronics: Automotive applications," Handbook of Flexible Organic Electronics: Materials, Manufacturing and Applications, 345, 2014, https://doi.org/10.1016/b978-1-78242-035-4.00014-2

[8] R. Anwar, L. Mao, H. Ning, R. Atakan, H.A. Tufan, S. uz Zaman, C. Cochrane, S.K. Bahadir, V. Koncar, F. Kalaoglu, B.A. Auld, C.E. Baum, D. Betancourt, K. Haase, A. Hübler, F. Ellinger, A.T. Blischak, M. Manteghi, A. Čolaković, L. Corchia, G. Monti, E. De Benedetto, A. Cataldo, L. Angrisani, P. Arpaia, L. Tarricone, F. Costa, S. Genovesi, A. Monorchio, et al., "The internet of things: How the next evolution of the internet is changing everything," IEEE Transactions on Antennas and Propagation, 59(12), 1-4, 2014, https://doi.org/10.7551/mitpress/10277.003.0004

[9] L. Corchia, G. Monti, E. De Benedetto, A. Cataldo, L. Angrisani, P. Arpaia, L. Tarricone, "Fully-Textile, Wearable Chipless Tags for Identification and Tracking Applications," Sensors, 20(2), 429, 2020 , https://doi.org/10.3390/s20020429

[10] L. Corchia, G. Monti, L. Tarricone, "Durability of wearable antennas based on nonwoven conductive fabrics: Experimental study on resistance to washing and ironing," International Journal of Antennas and Propagation, 2018, 2018, https://doi.org/10.1155/2018/2340293

[11] R. Atakan, H.A. Tufan, S. uz Zaman, C. Cochrane, S.K. Bahadir, V. Koncar, F. Kalaoglu, "Protocol to assess the quality of transmission lines within smart textile structures," Measurement, 152, 107194, 2020, https://doi.org/10.1016/j.measurement.2019.107194

[12] Y. Wang, L. Li, B. Wang, L. Wang, "A body sensor network platform for in-home health monitoring application," in Proceedings of the 4th International Conference on Ubiquitous Information Technologies \& Applications, IEEE: 1-5, 2009, https://doi.org/10.1109/icut.2009.5405731

[13] K. Finkenzeller, RFID handbook: fundamentals and applications in contactless smart cards, radio frequency identification and near-field communication, John Wiley \& Sons, 2010, https://doi.org/10.1002/9780470665121

[14] D.K. Klair, K.-W. Chin, R. Raad, "A survey and tutorial of RFID anticollision protocols," IEEE Communications Surveys \& Tutorials, 12(3), 400-421, 2010, https://doi.org/10.1109/surv.2010.031810.00037

[15] R. Rezaiesarlak, M. Manteghi, CHIPLESS RFID, Springer, 2016, https://doi.org/10.1007/978-3-319-10169-9_3

[16] J.P. Vinding, Interrogator-responder identification system, 1969.

[17] O.E. Rittenbach, Communication by radar beams, 1969, https://doi.org/10.1002/sce.3730530262

[18] R.M. Richardson, Remotely actuated radio frequency powered devices, 1963.

[19] R.F. Harrington, "Field measurements using active scatterers (correspondence)," IEEE Transactions on Microwave Theory and Techniques, 11(5), 454-455, 1963, .https://doi.org/10.1109/tmtt.1963.1125707

[20] H. Khaleel, Innovation in wearable and flexible antennas, Wit Press, 2014, https://doi.org/10.2495/978-1-84564-986-9/008

[21] D.P. Morgan, "History of SAW devices," in Proceedings of the 1998 IEEE International Frequency Control Symposium (Cat. No. 98CH36165), IEEE: 439-460, 1998, https://doi.org/10.1109/freq.1998.717937

[22] В.A. Auld, Acoustic fields and waves in solids, Рипол Классик, 1973.

[23] V.D. Hunt, A. Puglia, M. Puglia, RFID: a guide to radio frequency identification, John Wiley \& Sons, 2007.

[24] J. Foroughi, T. Mitew, P. Ogunbona, R. Raad, F. Safaei, "Smart Fabrics and Networked Clothing: Recent developments in CNT-based fibers and their continual refinement," IEEE Consumer Electronics Magazine, 5(4), 105111, 2016, https://doi.org/10.1109/mce.2016.2590220

[25] N.C. Karmakar, R. Koswatta, P. Kalansuriya, E. Rubayet, Chipless RFID reader architecture, Artech House, 2013.

[26] D. Betancourt, K. Haase, A. Hübler, F. Ellinger, "Bending and folding effect study of flexible fully printed and late-stage codified octagonal chipless RFID tags," IEEE Transactions on Antennas and Propagation, 64(7), 28152823, 2016, https://doi.org/10.1109/tap.2016.2559522

[27] A.C. Durgun, C.A. Balanis, C.R. Birtcher, D.R. Allee, "Design, simulation, fabrication and testing of flexible bow-tie antennas," IEEE Transactions on Antennas and Propagation, 59(12), 4425-4435, 2011, https://doi.org/10.1109/tap.2011.2165511

[28] F. Costa, S. Genovesi, A. Monorchio, "A chipless RFID based on 763 
multiresonant high-impedance surfaces," IEEE Transactions on Microwave Theory and Techniques, 61(1), 146-153, 2013, https://doi.org/10.1109/tmtt.2012.2227777

[29] M. Martinez, D. van der Weide, "Compact slot-based chipless RFID tag," in 2014 IEEE RFID Technology and Applications Conference (RFID-TA), IEEE: 233-236, 2014, https://doi.org/10.1109/rfid-ta.2014.6934234

[30] S. Rauf, M.A. Riaz, H. Shahid, M.S. Iqbal, Y. Amin, H. Tenhunen, "Triangular loop resonator based compact chipless RFID tag," IEICE Electronics Express, 14.20161262, 2017, https://doi.org/10.1587/elex.14.20161262

[31] M.A. Riaz, H. Shahid, S.Z. Aslam, Y. Amin, A. Akram, H. Tenhunen, "Novel T-shaped resonator based chipless RFID tag," IEICE Electronics Express, 14.20170728, 2017, https://doi.org/10.1587/elex.14.20170728

[32] R. Anwar, L. Mao, H. Ning, "Frequency selective surfaces: a review," Applied Sciences, 8(9), 1689, 2018, https://doi.org/10.3390/app8091689

[33] A. Blischak, M. Manteghi, "Pole residue techniques for chipless RFID detection," in 2009 IEEE Antennas and Propagation Society International Symposium, IEEE: 1-4, 2009, https://doi.org/10.1109/aps.2009.5172097

[34] A.T. Blischak, M. Manteghi, "Embedded singularity chipless RFID tags," IEEE Transactions on Antennas and Propagation, 59(11), 3961-3968, 2011, https://doi.org/10.1109/tap.2011.2164191

[35] R. Rezaiesarlak, M. Manteghi, "Complex-natural-resonance-based design of chipless RFID tag for high-density data," IEEE Transactions on Antennas and Propagation, 62(2), 898-904, 2013, https://doi.org/10.1109/tap.2013.2290998

[36] C.E. Baum, The singularity expansion method, Springer: 129-179, 1976, https://doi.org/10.1007/3540075534_8

[37] J.-M. Jin, Theory and computation of electromagnetic fields, John Wiley \& Sons, 2011, https://doi.org/10.1002/9780470874257

[38] M.U.A. Khan, R. Raad, J. Foroughi, F. Tubbal, P.I. Theoharis, M.S. Raheel, "Effects of Bending Bow-Tie Chipless RFID Tag for Different Polymer Substrates," in 2019 13th International Conference on Signal Processing and Communication Systems (ICSPCS), IEEE: 1-4, 2019, https://doi.org/10.1109/icspcs47537.2019.9008594

[39] A. Vena, E. Perret, S. Tedjini, "High-capacity chipless RFID tag insensitive to the polarization," IEEE Transactions on Antennas and Propagation, 60(10), 4509-4515, 2012, https://doi.org/10.1109/tap.2012.2207347

[40] I.Y. Immoreev, "-Signal Waveform Variations in Ultrawideband Wireless Systems: Causes and Aftereffects," Ultrawideband Radar: Applications and Design, 71-104, 2012, https://doi.org/10.1201/b12356-4 